\title{
PRR11 Overexpression Facilitates Ovarian Carcinoma Cell Proliferation, Migration, and Invasion Through Activation of the PI3K/AKT/ $\beta$-Catenin Pathway
}

\author{
Jing Zhu ${ }^{a}$ Hua Hua Jing Wang ${ }^{b}$ Ying Yang ${ }^{a}$ Ping Yib \\ ${ }^{a}$ Department of Gynaecology and Obstetrics, The Second Affiliated Xinqiao Hospital of Army Medical \\ University, Chongqing, bDepartment of Gynaecology and Obstetrics, The Third Affiliated Daping \\ Hospital of Army Medical University, Chongqing, China
}

\section{Key Words}

PRR11 - Ovarian carcinoma - Proliferation - Migration and invasion - PI3K/AKT/ß-catenin signaling

\begin{abstract}
Background/Aims: Ovarian cancer (OC) is the most lethal gynecologic malignancy, mainly due to the advanced stage at diagnosis in most patients and high rate of relapse. Thus, it is still essential to elucidate the underlying mechanisms and explore the diagnostic and therapeutic targets of OC. Recent studies have revealed that proline-rich protein 11 (PRR11) is dysregulated in different cancers, participating in their initiation and progression; however, it remains unclear whether PRR11 is involved in OC. Methods: Immunohistochemical staining, quantitative reverse transcription $P C R$, and western blotting were performed to evaluate PRR11 expression in OC tissues and cells. The relationship between PRR11 expression and the clinicopathologic data of patients were analyzed. We upregulated and downregulated PRR11 expression using a PRR11 overexpression vector and PRR11-specific small interfering RNA, respectively, to further clarify its role in the malignant biological behavior of $O C$ in vitro. Results: Overexpression of PRR11 in OC tissues and cells significantly correlated with advanced FIGO stage, lymph node metastasis, and large tumor size. Downregulation of PRR11 inhibited cell proliferation and prevented the invasion and migration of $\mathrm{HO}-8910 \mathrm{OC}$ cells, whereas opposite results were observed in Caov3 cells upon PRR11 upregulation. Further analyses showed that PRR11 positively regulated cell proliferation-related proteins, including c-myc and cyclin D1, and increased and decreased the expression of matrix metalloproteinase 2 and tissue inhibitor of metalloproteinase 2 , respectively. Additionally, our preliminary results demonstrated that PRR11 expression was mediated by the phosphoinositide 3-kinase/AKT/ $\beta$ -
\end{abstract}

\footnotetext{
Ping Yi Dept. of Gynaecology and Obstetrics, The Third Affiliated Daping Hospital of Army Med. Univ., Chongqing 400042 and Ying Yang (China); Dept. of Gynaecology and Obstetrics, The Second Affiliated Xinqiao Hospital of Army Med. Univ., Chongqqing 400037 (China); E-Mail yping012@163.com; yingy63@126.com
}

\section{KARGER}


catenin signaling pathway. Conclusion: The results of this study provide evidence that PRR11 plays a critical role in the progression and metastasis of $O C$, and as such, may serve as a potential prognostic and therapeutic target in OC.

\section{Introduction}

Ovarian cancer (OC) is the most common malignancy in women, and its incidence is increasing worldwide [1]. Despite the development of surgery and adjuvant therapies, the overall prognosis of patients with $\mathrm{OC}$ remains poor, especially in those with advanced stage disease [2]. At the molecular level, a series of genetic and epigenetic alterations that inactivate tumor suppressor genes and activate oncogenes play important roles in the pathogenesis of OC. Thus, identifying effective biomarkers for screening and molecular targeting is important for improving the prognosis of this disease.

Proline-rich protein 11 (PRR11) is located on human chromosome 17q22 region, and plays a vital role in numerous biological processes such as cell cycle progression, apoptosis, and invasion [3]. In recent years, accumulating evidence has shown that PRR11 is a candidate oncogene in mammals, is widely expressed in solid tumors, including pancreatic [4], lung [5], and hilar cholangiocarcinoma [6], and is closely related to local tumor recurrence and metastasis. Specific short hairpin RNA-mediated depletion of PRR11 causes cell cycle arrest, leading to a significant decrease in cell viability [7]. PRR11 has been regarded as a potential new target for the diagnosis and treatment of lung cancer, as it regulates key genes associated with tumor occurrence [8,9]. However, despite growing evidence demonstrating that PRR11 is as an important cancer-related protein, the role of PRR11 in OC and the underlying molecular mechanisms have not been investigated.

We assessed PRR11 expression in both OC tumor tissues and cell lines to investigate its effects on cell proliferation, invasion, and migration. We demonstrated that PRR11 was highly expressed in human OC tissues and enhanced the proliferation, migration, and invasion of OC cell lines in vitro, possibly by activating the phosphoinositide 3 -kinase (PI3K)/AKT/ $\beta$ catenin signaling pathway. These data provide significant insights into the role of PRR11 and its underlying molecular mechanisms in OC.

\section{Materials and Methods}

Tissue specimens

Fifty-one pairs of tumor and normal ovarian surface epithelium specimens were collected from patients with OC and other benign gynecological diseases who had undergone surgery at Xinqiao Hospital, Army Medical University (Chongqing, China) between 2015 and 2016, after they provided signed informed consent. All specimens were immediately snap-frozen in liquid nitrogen and maintained at $-80^{\circ} \mathrm{C}$ until use. The patients' clinical characteristics such as age, tumor size, International Federation of Gynecology and Obstetrics (FIGO) stage, lymph node status, and histological grade were collected for statistical analysis. Ethical approval for the study was approved by the Research Ethics Committee of Xinqiao Hospital.

\section{Immunohistochemistry}

For immunohistochemical analysis of PRR11 expression, 4- $\mu$ m tissue microarray sections obtained from all cancerous and non-cancerous tissues were paraffin-embedded and cut to construct tissue microarrays. The primary antibody anti-PRR11 (1:100; Abcam Inc., Cambridge, MA) was used for immunohistochemistry according to the manufacturer's instructions. Slides were routinely treated for deparaffinization and hydration, and then heated in $0.01 \mathrm{mM}$ citrate buffer (pH 6.0). Endogenous peroxidase activity was blocked in $3 \%$ hydrogen peroxide in methanol for $20 \mathrm{~min}$ at room temperature. Immunohistochemical signals were calculated by quantifying positively stained cells under a microscope (Olympus Corporation, Tokyo, Japan). Two histopathologists were blindly reviewed the slides and scored the staining. 


\section{Cellular Physiology Cell Physiol Biochem 2018;49:696-705 \begin{tabular}{ll|l} 
DOI: 10.1159/000493034 & $\begin{array}{l}\text { O 2018 The Author(s). Published by S. Karger AG, Basel } \\
\text { www.karger.com/cpb }\end{array}$
\end{tabular} \\ Zhu et al.: Role of PRR11 in Ovarian Cancer}

Cell culture

Four human ovarian cancer cell lines (Caov3, SKOV3, OVCAR3, and HO-8910) and a normal ovarian epithelial cell line IOSE80 were purchased from the cell bank of the Shanghai Biology Institute, Chinese Academy of Science (Shanghai, China). Cells were maintained at $37^{\circ} \mathrm{C}$ in a humidified atmosphere of $5 \% \mathrm{CO}_{2}$ in RPMI-1640 medium (Gibco, Gaithersburg, MA) supplemented with 10\% fetal bovine serum (FBS; Gibco), $100 \mathrm{U} / \mathrm{mL}$ penicillin, and $100 \mu \mathrm{g} / \mathrm{mL}$ streptomycin (Sigma, St. Louis, MO).

\section{Quantitative reverse transcription $P C R$}

Total RNA was extracted using the E.Z.N.A. Total RNA Kit I (Omega Bio-tek, Norcross, GA) according to the manufacturer's instructions. GAPDH expression served as the internal control. The following primers were used: PRR11 forward primer: 5'-CGTATCTGCCACCGAGAACTT-3' and reverse primer: 5'-GAGATGGTCTTCAGTGCTTC CT-3'; GAPDH forward primer: 5'-TGACTTCAACAGCGACACCCA-3' and reverse primer: 5'-CACCCTGTT GCTGTAGCCAAA-3'. Quantitative reverse transcription PCR (qRT-PCR) was performed using the SYBR Premix Ex Taq II (Takara), and the reaction conditions were: $95^{\circ} \mathrm{C}, 10 \mathrm{~min} ; 95^{\circ} \mathrm{C}$, $1 \mathrm{~min}, 55^{\circ} \mathrm{C}, 30 \mathrm{~s}$, and $72^{\circ} \mathrm{C}, 45 \mathrm{~s}$ (39 cycles).

\section{Cell transfection}

Small interfering RNA (siRNA; 5'-ACGCAGGCCUUAAGGAGAATT-3') for PRR11 was purchased from GenePharma (Shanghai, China). To generate PRR11 overexpression vectors, PRR11-coding sequences were obtained by qRT-PCR and cloned into the pEGFP-N1 vector. The resulting plasmid was designated pEGFP-N1-PRR11 (PRR11-EGPF). Caov3 cells were transfected with Lipofectamine ${ }^{\mathrm{TM}}$ RNAiMAX (Invitrogen, Carlsbad, CA) according to the manufacturer's instructions.

\section{Cell proliferation assay}

Cell proliferation was measured using the Cell Counting Kit 8 (CCK-8) assay (Dojindo, Kumamoto, Japan). Briefly, $24 \mathrm{~h}$ after transfection, $3 \times 10^{3}$ cells per well were plated into 96 -well plates and cultured in a humidified $37^{\circ} \mathrm{C}, 5 \% \mathrm{CO}_{2}$ incubator for up to 4 days. Then, $10 \mu \mathrm{L}$ CCK-8 reagent was added to each well, and the absorbance at $450 \mathrm{~nm}$ was measured using a microplate reader. The experiment was repeated three times.

\section{Colony formation assay}

For the colony formation assay, cells were transfected for $48 \mathrm{~h}$, plated into three 6 -cm cell culture dishes $\left(2 \times 10^{3}\right.$ cells per dish), and incubated at $37^{\circ} \mathrm{C}$. After 2 weeks, the plates were washed with phosphatebuffered saline and stained with crystal violet dye. Visible colonies were manually counted under a microscope. The experiments were performed three times in duplicate.

\section{Transwell migration and invasion assays}

For the Transwell assay, $5 \times 10^{4}$ transfected cells in $150 \mu \mathrm{L}$ RPMI 1640 medium without FBS were added to the upper chamber (BD Biosciences, San Jose, CA) or upper chamber precoated with $60 \mu \mathrm{L}$ matrigel (1:8 dilution; BD Biosciences). The lower chamber was filled with $600 \mu \mathrm{L}$ RPMI 1640 containing 15\% FBS. Then, cells were cultured at $37^{\circ} \mathrm{C}$ in a $5 \% \mathrm{CO}_{2}$ atmosphere. After the cells were incubated for $24 \mathrm{~h}$, the cells remaining on the upper membrane were removed with cotton wool, and the cells adhering to the lower surface were fixed in $4 \%$ methanol and dyed with $1 \%$ crystal violet for $30 \mathrm{~min}$. The cells that migrated or invaded through the membrane were imaged and counted using a microscope (Olympus); the experiments were repeated three times.

\section{Western blot analysis}

Western blot analysis was performed as previously described [10]. Total proteins from OC tissues and each group of cells were harvested and lysed with RIPA buffer (Beyotime, Beijing, China). Proteins were detected with anti-PRR11, anti- $\beta$-catenin (Abcam Inc.), anti-cyclin D1, anti-c-myc, anti-matrix metalloproteinase 2 (MMP2), anti-tissue inhibitor of metalloproteinase 2 (TIMP-2), anti-AKT, and antiphosphorylated (p-AKT) (Cell Signaling Technology, Danvers, MA). The secondary antibody used was goat anti-rabbit IgG horseradish peroxidase conjugate (1:3000; Cell Signaling Technology). 


\section{Cellular Physiology Cell Physiol Biochem 2018;49:696-705 \begin{tabular}{ll|l} 
DOI: 10.1159/000493034 & $\begin{array}{l}\text { O } 2018 \text { The Author(s). Published by S. Karger AG, Basel } \\
\text { wwwwkarger.com/cpb }\end{array}$
\end{tabular}

\begin{abstract}
Statistical analysis
Statistical analyses were performed with GraphPad Prism 5 software. All data are expressed as the mean \pm standard deviation and determined by the $t$-test. The chisquared test was used to examine possible correlations between PRR11 expression and clinicopathologic factors. $P$ values less than 0.05 were considered statistically significant.
\end{abstract}

\section{Results \\ Correlation between PRR11 upregulation with clinicopathological factors in OC \\ To assess the protein} levels of PRR11 in OC tissues, immunohistochemistry staining of PRR11 was performed. The results showed that PRR11 protein was mainly located in the cytoplasm, and PRR11 expression was high in 34 of 51 OC tissues (Fig. 1A). We also analyzed the expression levels of PRR11 in 51 snap-frozen OC tissues and normal ovarian surface epithelium using qRTPCR and westernblotting. PRR11 mRNA and protein expression was clearly increased in OC tissues compared with normal tissues (Fig. 1B, C). We divided PRR11 protein levels into high expression and low expression groups according to immunohistochemistry staining results, and the correlation between PRR11 expression and clinicopathological parameters of OC was analyzed. The results demonstrated that the upregulation of PRR11 expression was positively correlated with advanced FIGO stage (III/IV) and large tumor size. In condition, PRR11 expression was higher in tumors with positive lymph node metastasis compared with those without metastasis. However, no statistical significance was found between PRR11 levels and patient age and histological grade (Table 1). Collectively, these data showed that

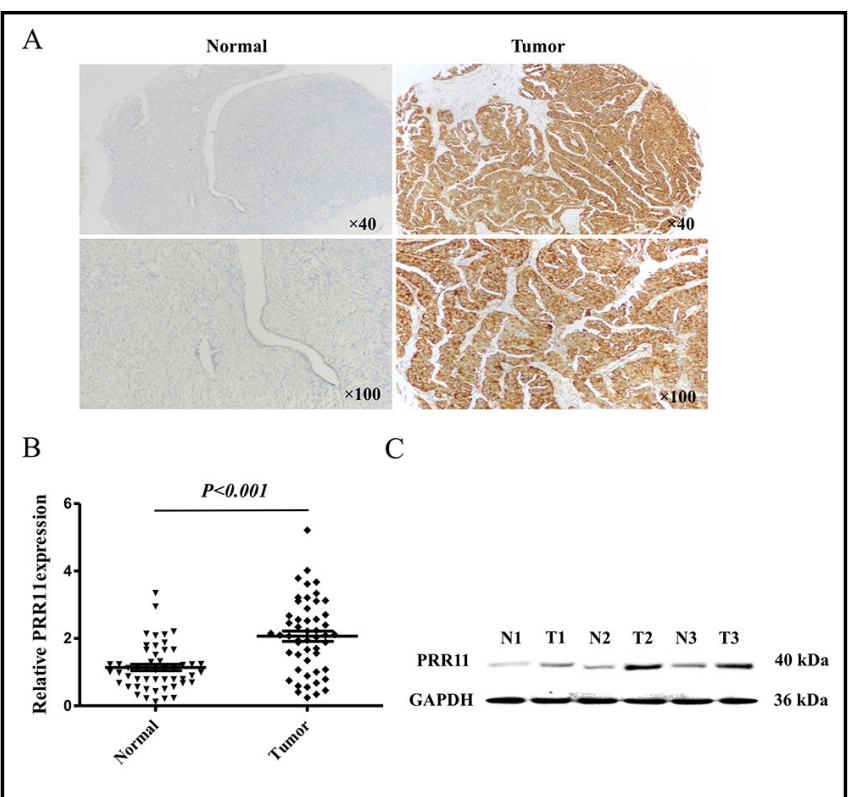

Fig. 1. Expression of PRR11 in OC tissue specimens. (A) Representative images of immunostained OC and normal ovarian tissues showing levels of PRR11 protein expression $(n=51)$. (B) PRR11 mRNA transcript levels in OC tissue and normal specimens ( ${ }^{* * *} \mathrm{P}<0.001$ vs. normal). GAPDH was detected as the internal control. (C) Western blot analysis of three randomly selected cases of OC (T) and normal ovarian $(\mathrm{N})$ tissues showing protein levels of PRR11 and GAPDH.

Table 1. Correlation between PRR11 expression and clinicopathologic variables in OC patients $(n=51) .{ }^{*} p<$ 0.05 was considered significant

\begin{tabular}{|c|c|c|c|c|c|}
\hline \multirow{3}{*}{ Parameters Total } & \multirow{3}{*}{ Group } & \multirow{3}{*}{ Total } & \multicolumn{2}{|c|}{ PRR11 expression } & \multirow{3}{*}{$P$ value } \\
\hline & & & & & \\
\hline & & & High & low & \\
\hline \multirow{3}{*}{ Age (years) } & $<50$ & 13 & 8 & 5 & \multirow{3}{*}{0.649} \\
\hline & & & & & \\
\hline & $\geq 50$ & 38 & 26 & 12 & \\
\hline \multirow{3}{*}{ FIGO stage } & I/II & 14 & 6 & 8 & \multirow{3}{*}{$0.026^{*}$} \\
\hline & & & & & \\
\hline & III/IV & 37 & 28 & 9 & \\
\hline \multirow{3}{*}{ lymph node metastasis } & Negative & 25 & 13 & 12 & \multirow{3}{*}{$0.029^{*}$} \\
\hline & & & & & \\
\hline & Positive & 26 & 21 & 5 & \\
\hline \multirow{3}{*}{ Histological grade } & G1-G2 & 14 & 11 & 3 & \multirow{3}{*}{0.267} \\
\hline & & & & & \\
\hline & G3 & 37 & 23 & 14 & \\
\hline \multirow{3}{*}{ Tumor size $(\mathrm{cm})$} & $\geq 4$ & 31 & 24 & 7 & \multirow{3}{*}{$0.042^{*}$} \\
\hline & & & & & \\
\hline & $<4$ & 20 & 10 & 10 & \\
\hline
\end{tabular}


PRR11 is overexpressed in OC, and that the level of PRR11 expression may play a critical role in the occurrence and progression of $\mathrm{OC}$.

PRR11 expression in OC cell lines

Western blotting was performed to evaluate the expression of PRR11 in OC cell lines. PRR11 protein expression was increased in OC cell lines compared with the ovarian epithelial cell line IOSE80 (Fig. 2A). To further clarify the effects of PRR11 on the malignant biological behaviors of OC in vitro, we used a PRR11 overexpression vector and PRR11-specific siRNAs to perform PRR11 gain-of-function and loss-of-function studies in OC cells. Then, qRT-PCR and western blot analysis were performed to determine the efficiency of PRR11 expression. The results suggested that PRR11 expression in the siRNAPRR11 group was significantly lower than that in the siRNAnegative control (NC) group (Fig. 2B). Compared with the control group, the mRNA and protein expression of PRR11 significantly increased in the PRR11-EGFP group (Fig. 2C).

\section{PRR11 regulates OC cell proliferation in vitro}

To investigate the role of PRR11 in OC progression, the CCK-8 and colony formation assays were performed. As shown in Fig. 3A, PRR11 silencing in HO-8910 cells significantly inhibited cell growth compared with siRNA-NC cells, and the number of colonies was significantly reduced (Fig. 3B). Conversely, a higher growth rate and enhanced clonogenic capacity were revealed in the PRR11-EGFP group compared with the vector group (Fig. 3C, D). These data provide evidence that PRR11 may function as a positive regulator of cell proliferation.

\section{PRR11 regulates OC cell migration and invasion in vitro}

Because cell migration and invasion are essential prerequisites for cancer metastasis, the Transwell assay was performed to evaluate the effect of differential PRR11 expression on cell migration and invasion in OC cell lines. The results showed that the depletion of PRR11 led to significant reduction of the invasion and migration abilities of HO-8910 cells (Fig. 4A), whereas the upregulation of PRR11 expression rescued the metastatic phenotype in Caov3 cells (Fig. 4B). 
Fig. 3. Effects of PRR11 on OC cell proliferation. (A) Proliferation of PRR11-silenced HO-8910 cells was measured by the CCK-8 assays at the indicated number of days ( $\mathrm{n}=3,{ }^{*} \mathrm{P}<0.05$ vs. NC group). (B) Quantitative data from the colony formation assay and representative images of colonies by HO-8910 cells 2 weeks post-transduction with siRNA-targeting PRR11 or NC $(n=3$, ${ }^{*} \mathrm{P}<0.05$ vs. NC group). (C) CCK-8 assay showing that upregulation of PRR11 promoted the proliferation of Caov3 cells ( $\mathrm{n}=3,{ }^{*} \mathrm{P}<0.05$ vs. vector group). (D) Colony formation assays showed that upregulation of PRR11 significantly enhanced the colony-forming ability of Caov3 cells compared with control cells $(n=3$, ${ }^{*} \mathrm{P}<0.05$ vs. vector group).
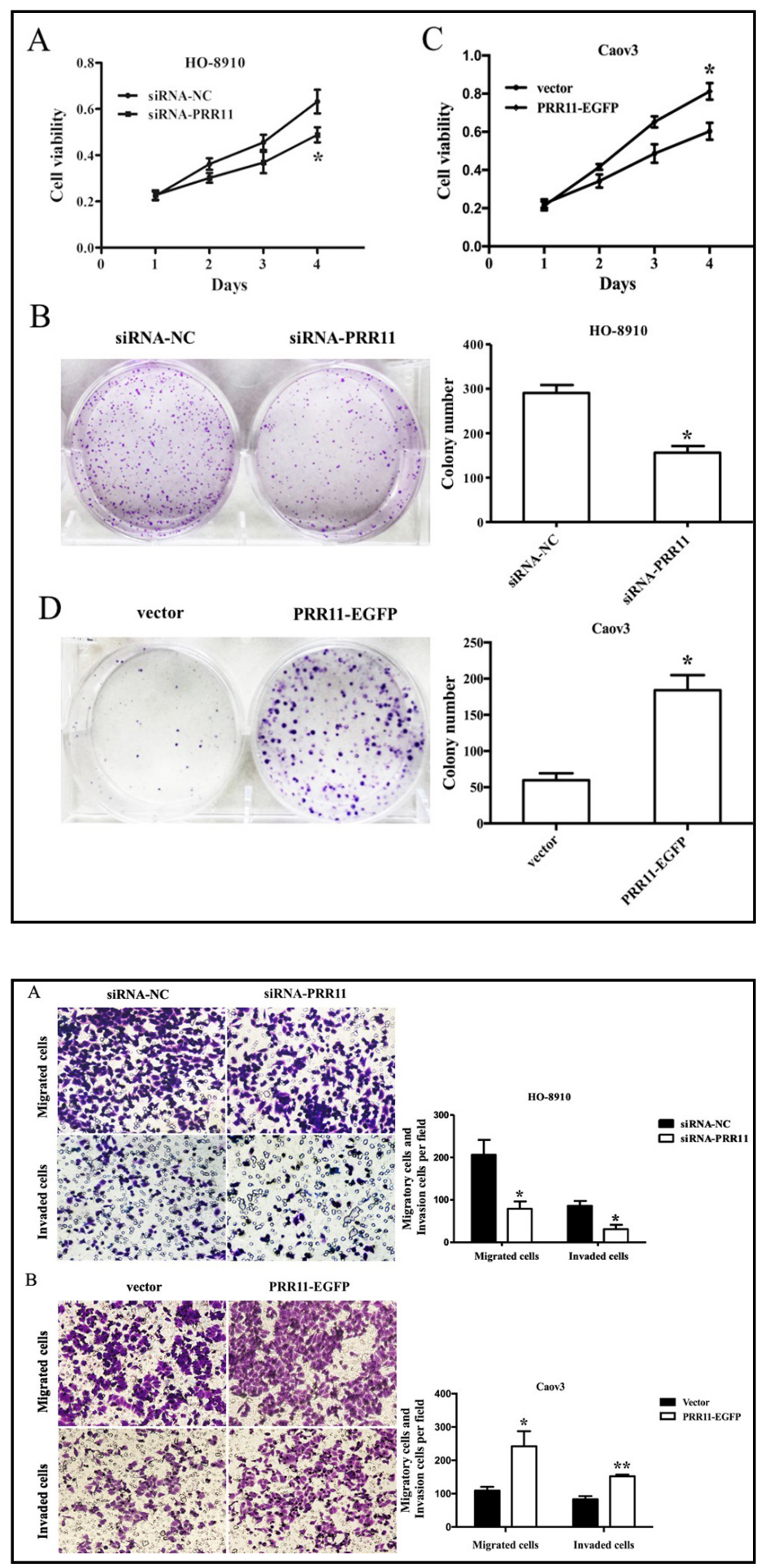

Fig. 4. Effects of PRR11 on the cell migration and invasion of OC cell lines. (A) Representative images and quantitative analysis showing inhibited migration and invasion of HO-8910 cells after PRR11-targeting siRNA transfection as determined by the Transwell assay (Original magnification $200 \times, n=3,{ }^{*} \mathrm{P}<0.05$ vs. NC group). (B) Representative images and quantitative analysis showing promoted migration and invasion of Caov3 cells after pEGFPN1-PRR11 plasmid transfection as determined by the Transwell assay (original magnification $200 \times, n=3$, ${ }^{*} \mathrm{P}<0.05,{ }^{* *} \mathrm{P}<0.01$ vs. vector group).

\section{Expression of c-myc, cyclin D1, MMP2, and TIMP-2 mediated by PRR11}

To further investigate the potential mechanism of PRR11-induced cell proliferation, changes in the expression of several proliferation-related proteins were examined. Western blot analysis indicated that the levels of c-myc and cyclin D1 were significantly downregulated after PRR11-siRNA transfection (Fig. 5A), but were significantly increased in the PRR11 overexpression group compared to the respective corresponding controls (Fig. 5B). These results suggested that PRR11 contributed to OC cell proliferation, partly by affecting the 
expression of c-myc and cyclin D1. MMP2 and TIMP-2 are thought to play important roles in mediating tumor cell metastasis [11]. Activated MMP2 degrades extracellular matrix (ECM) components to allow cancer cell expansion without resistance, whereas TIMP-2 is a specific inhibitor of MMP2 [12]. In this study, we validated that PRR11 could regulate the expression of MMP2 and TIMP-2 in two OC cell lines. Compared with the corresponding controls, MMP2 expression was greatly downregulated in $\mathrm{HO}$ 8910 cells, whereas TIMP-2 expression was dramatically upregulated after PRR11 knockdown (Fig. 5C). On the other hand, opposite results were observed in PRR11enhanced Caov3 cells (Fig. 5D). These results indicate that PRR11 may control OC cell migration and invasion by regulating the expression of MMP2 and TIMP-2.

Mechanism by which PRR11 exerts its functions

To investigate the downstream mechanisms underlying the role of PRR11 in OC progression, we investigated the PI3K/AKT signaling pathway. Western blot analysis demonstrated that PRR11 silencing decreased p-AKT and cytoplasmic and nuclear $\beta$-catenin expression in $\mathrm{HO}$ 8910 cells (Fig. 6A), whereas restoration of PRR11 had the opposite effects in Caov3 cells (Fig. 6B). A specific inhibitor of PI3K/AKT (LY294002) was added to the Caov3 cells with PRR11 overexpression, and the results showed that the levels of p-AKT and expression of cytoplasmic and nuclear $\beta$-catenin were reversed.

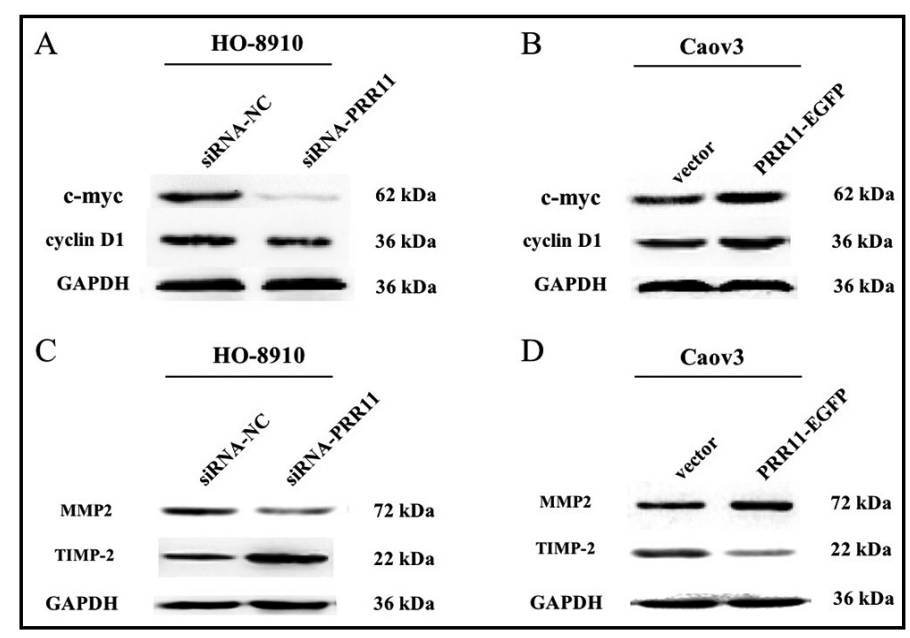

Fig. 5. PRR11 regulates OC proliferation and migration/invasionrelated proteins in OC cells. (A) PRR11 knockdown inhibited the expression of c-myc and cyclin D1 in the siRNA-PRR11 group of HO-8910 cells. (B) PRR11 upregulation enhanced the expression of c-myc and cyclin D1 in the PRR11-EGFP group of Caov3 cells. (C) Silencing of PRR11 expression decreased MMP2 expression and increased TIMP-2 expression in HO-8910 cells. (D) Overexpression of PRR11 upregulated MMP2 and downregulated TIMP-2 expression in Caov3 cells.

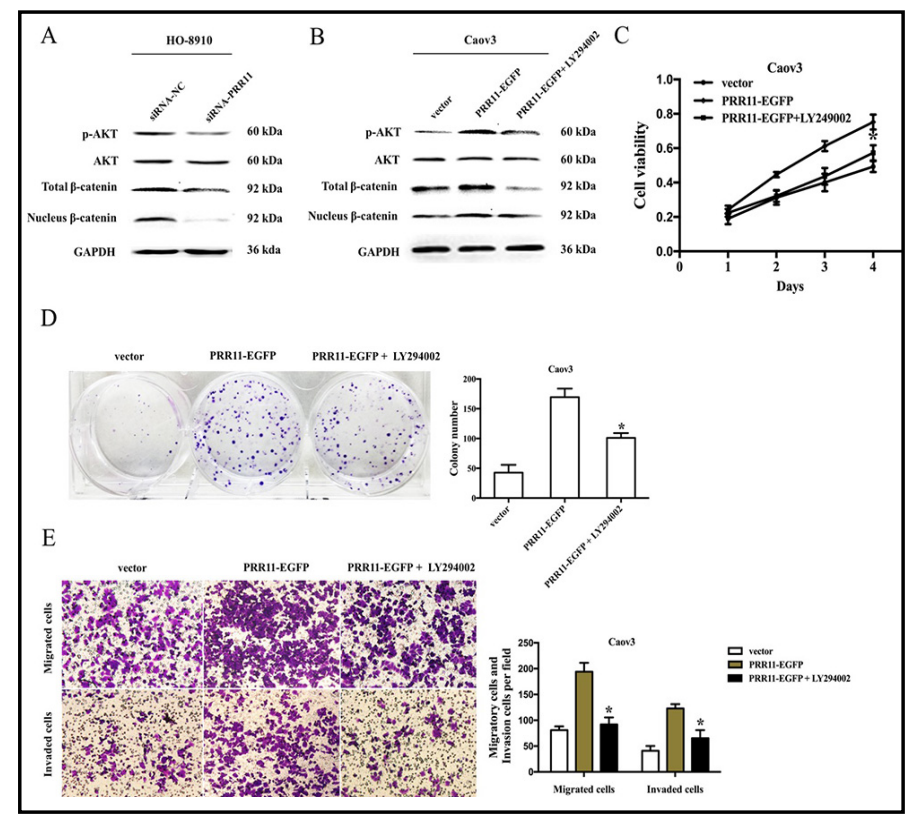

Fig. 6. Effects of PRR11 on the PI3K/AKT signaling pathway. (A) After being transfected with PRR11-siRNA, the expression of p-AKT/AKT and $\beta$-catenin was analyzed by western blotting in HO-8910 cells. (B) LY294002 was added to PRR11-overexpressing Caov3 cells, and the expression of $\mathrm{p}-\mathrm{AKT} / \mathrm{AKT}$ and $\beta$-catenin was detected by western blotting, after which the proliferation $(\mathrm{C})$, clonogenic capacity (D). and migration/invasion (magnification $200 \times$ ) (E) of Caov3 cells was measured. The quantitative data are shown ( $\mathrm{n}=3,{ }^{*} \mathrm{p}<0.05$ vs. PRR11-EGFP group). 


\section{Cellular Physiology Cell Physiol Biochem 2018;49:696-705 \\ and Biochemistry Published \begin{tabular}{l|l} 
DOI: 10.1159/000493034 & $\begin{array}{l}\text { (c) } 2018 \text { The Author(s). Published by S. Karger AG, Basel } \\
\text { www.karger.com/cpb }\end{array}$
\end{tabular} \\ Zhu et al.: Role of PRR11 in Ovarian Cancer}

Consistent with this, the enhancement of cell proliferation, clonogenicity, and metastasis by PRR11 upregulation was inhibited by LY294002 exposure in Caov3 cells (Fig. 6C-E). These findings showed that activation of the PI3K/AKT/ $\beta$-catenin axis was essential for PRR11 to promote $\mathrm{OC}$ cell proliferation and migration/invasion.

\section{Discussion}

Previous studies have shown that PRR11 overexpression may be a hallmark of aggressiveness and recurrence in various types of cancer tissues and cell lines, participating in cell cycle progression and apoptosis through transcriptional regulation of related genes [13]. This was the first study to show that PRR11 is abundantly expressed in OC tissues and cell lines compared with those in non-cancerous tissues and the normal ovarian epithelial cell line IOSE80. Moreover, high PRR11 expression was significantly correlated with advanced FIGO stage, large tumor size, and lymph node metastasis of patients with OC. These data show that PRR11 functions as an oncogene in OC origin and development, although its precise role in this disease remains to be clarified.

Our subsequent studies showed that PRR11 silencing inhibited cell proliferation and caused a dramatic decrease in the colony formation of HO-8910 cells. These results were confirmed by upregulation of PRR11 in the Caov3 cell line. The CCK-8 assay showed that the growth of Caov3 cells transfected with the overexpression vector was increased compared with control cells. The results of the colony formation assay revealed that clonogenic survival was increased following the upregulation of PRR11 in Caov3 cells. These data suggest the anti-tumor action of PRR11 silencing. c-Myc, a nuclear phosphoprotein, is also a downstream effector of $\beta$-catenin that directly responds to mitogenic signals and functions as a transcription factor that stimulates the cell cycle and cancer progression [14,15]. Studies have revealed that cyclin D1 is implicated in the cell cycle progression of various tumors and is associated with the cell proliferative activity and differentiation of OC. Suppression of cyclin D1 is closely correlated with reduced tumorigenicity $[16,17]$. In this study, we examined the protein expression of c-myc and cyclin D1 by western blot analysis after PRR11 overexpression. The results showed that c-myc and cyclin D1 expression was significantly decreased in the siRNA-PRR11 group, whereas the opposite results were observed in Caov3 cells upon PRR11 upregulation. Together, these findings indicate that PRR11 may promote OC cell proliferation by upregulation cyclin D1 and c-myc expression via modulation of the cell cycle.

Cancer metastasis is associated with a poor prognosis in patients with OC, causes approximately $90 \%$ of cancer-related deaths, and is regarded as a series of interdependent events that include degradation of ECM and basement membrane components, which is a critical step in metastasis [18]. ECM degradation is positively mediated by MMP/TIMP expression, as alterations in the balance of MMP/TIMP expression lead to the invasion and migration of OC cells $[19,20]$. Here, we observed that PRR11 silencing mitigated the invasion and migration of siRNA-PRR11 HO-8910 cells, which was associated with reduced levels of MMP2, but increased levels of TIMP-2 in OC. In contrast, upregulation of PRR11 significantly increased the invasion and migration abilities of Caov3 cells. Western blot analysis further confirmed that upregulation of PRR11 increased the expression of MMP2 and inhibited the expression of TIMP-2 compared to the control group. These data suggest that PRR11 contributes to OC cells metastasis, partly by regulating the balance of MMP2/ TIMP-2 expression and controlling ECM degradation and remodeling.

PI3K/AKT, a major signaling pathway associated with cancer progression and metastasis, is aberrantly activated in OC, leading to recruitment and activation of the AKT serine/threonine kinase $[21,22]$. The PI3K/AKT pathway is a potential target for therapeutic intervention due to its pivotal role in the regulation of proliferation, survival, and invasiveness of OC [23]. Hematopoietic PBX-interacting protein promoted the migration, invasion, and EMT in OAW42 cells via activation of the PI3K/AKT pathway by directly interacting with 


\section{Cellular Physiology Cell Physiol Biochem 2018;49:696-705 \begin{tabular}{l|l|l} 
DOI: 10.1159/000493034 & $\begin{array}{l}\text { O } 2018 \text { The Author(s). Published by S. Karger AG, Basel } \\
\text { wwww.karger.com/cpb }\end{array}$
\end{tabular} \\ Zhu et al.: Role of PRR11 in Ovarian Cancer}

PI3K [24]. He et al. [25] revealed that exendin-4 inhibit cell growth, migration, and invasion and enhanced apoptosis by inhibiting the PI3K/AKT pathway during OC progression. Additionally, phosphorylation of $\beta$-catenin by AKT increases its transcriptional activity, leading to the accumulation of $\beta$-catenin in the cytosol and nucleus, and resulting in the activation and expression of downstream target genes, such as MMP2/TIMP-2, which causes cell invasiveness and metastasis [26, 27]. In condition, it was revealed that suppressing $\beta$-catenin resulted in the decreased expression of c-myc and cyclin D1, and induced antitumor growth effects in OC cells [28, 29].

In this study, we demonstrated that HO-8910 cells displayed decreased phosphorylation of AKT after PRR11 silencing, and both the transcriptional activity and nuclear distribution of its downstream critical regulator $\beta$-catenin also significantly decreased in these cells. By contrast, PRR11 upregulation caused significant upregulation of p-AKT, and cytoplasmic and nuclear $\beta$-catenin expression in Caov3 cells. These data suggest that PRR11 may exert its functions by enhancing the PI3K/AKT/ $\beta$-catenin-mediated signaling pathway. To confirm how the PI3K/AKT/ $\beta$-catenin axis is involved in the process of PRR11-mediated cell proliferation and metastasis, LY294002, a specific inhibitor of PI3K, was added to PRR11overexpressing Caov3 cells, after which cell proliferation and migration/invasion were markedly abrogated. These findings demonstrated that the PI3K/AKT/ $\beta$-catenin signaling pathway contributed to the PRR11-mediated promotion of proliferation and migration/ invasion of OC cells. However, in-depth mechanistic studies are needed on its signaling pathway based on the regulatory biologic behavior of OC.

The results of this study showed that PRR11 is highly expressed in OC cell lines and tissues, and is closely associated with advanced FIGO stage, lymph node metastasis, and large tumor size. PRR11 facilitated the cell proliferation and migration/invasion of OC cells, the effects of which were dramatically mediated by activation of the PI3K/AKT/ $\beta$-catenin pathway and modulation of cyclin D1, c-myc, MMP2, and TIMP-2 expression. Although further studies are needed, our data revealed that PRR11 may be an important oncoprotein and potential therapeutic target in OC.

\section{Disclosure Statement}

The authors have no conflicts of interest to declare.

\section{References}

1 Bruggmann D, Pulch K, Klingelhofer D, Pearce CL, Groneberg DA: Ovarian cancer: density equalizing mapping of the global research architecture. Int J Health Geogr 2017;16:3.

2 Zhao J, Pan Y, Li X, Zhang X, Xue Y, Wang T, Zhao S, Hou Y: Dihydroartemisinin and Curcumin Synergistically Induce Apoptosis in SKOV3 Cells Via Upregulation of MiR-124 Targeting Midkine. Cell Physiol Biochem 2017;43:589-601.

3 Ji Y, Xie M, Lan H, Zhang Y, Long Y, Weng H, Li D, Cai W, Zhu H, Niu Y, Yang Z, Zhang C, Song F, Bu Y: PRR11 is a novel gene implicated in cell cycle progression and lung cancer. Int J Biochem Cell Biol 2013;45:645-656.

-4 Tan S, Jiang Z, Hou A, Wang J, Zhang J, Dai L: Expression of PRR11 protein and its correlation with pancreatic cancer and effect on survival. Oncol Lett 2017;13:4117-4122.

$>5$ Zhao Q: RNAi-mediated silencing of praline-rich gene causes growth reduction in human lung cancer cells. Int J Clin Exp Pathol 2015;8:1760-1767.

6 Chen Y, Cha Z, Fang W, Qian B, Yu W, Li W, Yu G, Gao Y: The prognostic potential and oncogenic effects of PRR11 expression in hilar cholangiocarcinoma. Oncotarget 2015;6:20419-20433.

-7 Zhang C, Zhang Y, Li Y, Zhu H, Wang Y, Cai W, Zhu J, Ozaki T, Bu Y: PRR11 regulates late-S to G2/M phase progression and induces premature chromatin condensation (PCC). Biochem Biophys Res Commun 2015;458:501-508. 


\section{Cellular Physiology Cell Physiol Biochem 2018;49:696-705 \begin{tabular}{l|l|l} 
DOI: 10.1159/000493034 & $\begin{array}{l}\text { O 2018 The Author(s). Published by S. Karger AG, Basel } \\
\text { www.karger.com/cpb }\end{array}$ \\
\hline
\end{tabular}

8 Wang Y, Zhang Y, Zhang C, Weng H, Li Y, Cai W, Xie M, Long Y, Ai Q Liu Z, Du G, Wang S, Niu Y, Song F, Ozaki T, Bu Y: The gene pair PRR11 and SKA2 shares a NF-Y-regulated bidirectional promoter and contributes to lung cancer development. Biochim Biophys Acta 2015;1849:1133-1144.

-9 Song Z, Liu W, Xiao Y, Zhang M, Luo Y, Yuan W, Xu Y, Yu G, Hu Y: PRR11 Is a Prognostic Marker and Potential Oncogene in Patients with Gastric Cancer. PLoS One 2015;10:e0128943.

10 Wang M, Zhang Y, Wang T, Zhang J, Zhou Z, Sun Y, Wang S, Shi Y, Luan X, Zhang Y, Wang Y, Wang Y, Zou Z, Kang L, Liu H: The USP7 Inhibitor P5091 Induces Cell Death in Ovarian Cancers with Different P53 Status. Cell Physiol Biochem 2017;43:1755-1766.

11 Wang J, Ren Y, Guo X, Cheng H, Ye Y, Qi J, Yang C, You H: Alterations in enhancer of zeste homolog 2, matrix metalloproteinase- 2 and tissue inhibitor of metalloproteinase-2 expression are associated with ex vivo and in vitro bone metastasis in renal cell carcinoma. Mol Med Rep 2015;11:3585-3592.

12 Xu X, Wang Y, Chen Z, Sternlicht MD, Hidalgo M, Steffensen B: Matrix metalloproteinase-2 contributes to cancer cell migration on collagen. Cancer Res 2005;65:130-136.

13 Larance M, Ahmad Y, Kirkwood KJ, Ly T, Lamond AI: Global subcellular characterization of protein degradation using quantitative proteomics. Mol Cell Proteomics 2013;12:638-650.

14 Gordan JD, Thompson CB, Simon MC: HIF and c-Myc: sibling rivals for control of cancer cell metabolism and proliferation. Cancer Cell 2007;12:108-113.

15 Bretones G, Delgado MD, Leon J: Myc and cell cycle control. Biochim Biophys Acta 2015;1849:506-516.

16 Tashiro E, Tsuchiya A, Imoto M: Functions of cyclin D1 as an oncogene and regulation of cyclin D1 expression. Cancer Sci 2007;98:629-635.

17 Liang S, Mu K, Wang Y, Zhou Z, Zhang J, Sheng Y, Zhang T: CyclinD1, a prominent prognostic marker for endometrial diseases. Diagn Pathol 2013;8:138.

18 Yang JS, Lin CW, Hsin CH, Hsieh MJ, Chang YC: Selaginellatamariscina attenuates metastasis via Akt pathways in oral cancer cells. PLoS One 2013;8:e68035.

19 Roomi MW, Kalinovsky T, Rath M, Niedzwiecki A: Modulation of u-PA, MMPs and their inhibitors by a novel nutrient mixture in human female cancer cell lines. Oncol Rep 2012;28:768-776.

20 Yin X, Feng C, Han L, Ma Y, Jiao Y, Wang J, Jia L, Jing F, Gao X, Zhang Y, Zhang J: Diallyl disulfide inhibits the metastasis of type esophagealgastric junction adenocarcinoma cells via NF-kappaB and PI3K/AKT signaling pathways in vitro. Oncol Rep 2018;39:784-794.

21 Broussard JA, Rappaz B, Webb DJ, Brown CM: Fluorescence resonance energy transfer microscopy as demonstrated by measuring the activation of the serine/threonine kinase Akt. Nat Protoc 2013;8:265-281.

-22 Spangle JM, Roberts TM, Zhao JJ: The emerging role of PI3K/AKT-mediated epigenetic regulation in cancer. Biochim Biophys Acta 2017;1868:123-131.

23 Pons-Tostivint E, Thibault B, Guillermet-Guibert J: Targeting PI3K Signaling in Combination Cancer Therapy. Trends Cancer 2017;3:454-469.

-24 Bugide S, Gonugunta VK, Penugurti V, Malisetty VL, Vadlamudi RK, Manavathi B: HPIP promotes epithelialmesenchymal transition and cisplatin resistance in ovarian cancer cells through PI3K/AKT pathway activation. Cell Oncol (Dordr) 2017;40:133-144.

25 He W, Yu S, Wang L, He M, Cao X, Li Y, Xiao H: Exendin-4 inhibits growth and augments apoptosis of ovarian cancer cells. Mol Cell Endocrinol 2016;436:240-249.

-26 Karam AK, Santiskulvong C, Fekete M, Zabih S, Eng C, Dorigo O: Cisplatin and PI3kinase inhibition decrease invasion and migration of human ovarian carcinoma cells and regulate matrix-metalloproteinase expression. Cytoskeleton (Hoboken) 2010;67:535-544.

-27 Xia Y, Wu S: Tissue inhibitor of metalloproteinase 2 inhibits activation of the beta-catenin signaling in melanoma cells. Cell Cycle 2015;14:1666-1674.

28 Gwak H, Kim Y, An H, Dhanasekaran DN, Song YS: Metformin induces degradation of cyclin D1 via AMPK/ GSK3beta axis in ovarian cancer. Mol Carcinog 2017;56:349-358.

29 Zhai Y, Wu R, Schwartz DR, Darrah D, Reed H, Kolligs FT, Nieman MT, Fearon ER, Cho KR: Role of beta-catenin/ T-cell factor-regulated genes in ovarian endometrioid adenocarcinomas. Am J Pathol 2002;160:1229-1238. 